\title{
Dynamics of ternary liquid mixtures: Generalized collective modes analysis
}

\author{
T.M.Bryk ${ }^{1,2}$, I.M.Mryglod ${ }^{1,3}$ \\ ${ }^{1}$ Institute for Condensed Matter Physics of the National Academy of Sciences of Ukraine, 1 Svientsitskii Str., \\ 79011 Lviv, Ukraine \\ 2 Institute of Applied Mathematics and Fundamental Sciences, National Technical University of Lviv, 79013 \\ Lviv, Ukraine \\ 3 Institute for Theoretical Physics, Linz University, 4040 Linz, Austria
}

Received November 13, 2007

\begin{abstract}
A parameter-free generalized collective modes (GCM) approach is applied to the study of longitudinal and transverse dynamics in a ternary Lennard-Jones liquid mixture. Spectra of collective excitations are calculated in a wide range of wavenumbers starting from the hydrodynamic region and up to the range of molecular regime. Several simplified dynamical models are used in order to estimate the origin of branches in the spectra of collective excitations. This analysis permits to establish a crossover from a "coherent" to "partial" type of collective dynamics in the dispersion laws of propagating collective modes. The "coherent" type of collective dynamics is observed in the hydrodynamic region and is represented by sound modes (in the transverse case - shear waves) and propagating optic-like excitations, while in the range of "partial" dynamics (intermediate and large wavenumbers) the dynamical properties are mainly determined by correlated "partial" motions of particles of each species. Within the GCM scheme used the number of propagating optic-like modes, identified in this study, is simply related to the number of components in a multi-component mixture, and this has a close analogy in the dynamics of solids.
\end{abstract}

Key words: ternary liquid mixtures, collective excitations, optic-like modes, molecular dynamics

PACS: 05.20.Jj, 61.20.Ja, 61.20.LC

\section{Introduction}

Collective dynamics in disordered systems is one of the complex and unresolved problems in statistical physics. The existence of propagating and relaxation processes of different origin and having distinct characteristic times makes the collective dynamics in liquids extremely difficult to be analytically treated. Nevertheless, modern theoretical approaches of generalized hydrodynamics [1] to a certain extent make it possible to correctly describe hydrodynamic and non-hydrodynamic processes as well as the coupling effects between them in simple [2] and molecular liquids [3,4].

Over the last decade an essential advance was made in the theoretical description of collective dynamics in simple, binary and molecular liquids within the framework of the approach of generalized collective modes (GCM), which, like in hydrodynamics, results in decomposition of relevant time correlation functions into separated mode contributions coming from generalized hydrodynamic and non-hydrodynamic (kinetic) processes. The GCM approach consists in treating the collective dynamics on an extended basis set of $\mathrm{N}_{\mathrm{v}}$ dynamical variables, that apart from the densities of hydrodynamic variables contain their time derivatives, whose purpose is to correctly describe non-hydrodynamic short-time processes in liquids. Being consistent with the general definition of collective excitations in statistical physics as the poles of relevant Green functions, the GCM approach permits to calculate the dynamical eigenmodes in the system and to find their partial contributions to the time correlation functions. All the eigenmodes obtained within the GCM approach can be divided into two groups: (i) generalized hydrodynamic modes, having correct hydrodynamic asymptotes in the long-wavelength limit and the lifetime of which scales as $k^{-2}$ for $k \rightarrow 0$, and (ii) kinetic modes with a finite nonzero lifetime in the long-wavelength limit, 
being irrelevant to the dynamics on large spatial and temporal scales. Kinetic collective modes are important beyond the hydrodynamic region, where they essentially contribute to the shape of time correlation functions. In the cases of simple and binary liquids, the GCM studies [5-9] permit us to perform a classification of kinetic collective modes of different physical origin. As examples of kinetic propagating modes one can mention shear and heat waves, and for binary liquids there are found optic-like excitations that, by analogy with optic phonons in binary crystals, reflect the opposite-phase oscillations of the neighbors of different kind. A non-hydrodynamic process of structural relaxation is the most obvious example of kinetic relaxing modes, which are not taken into account within the standard hydrodynamics.

In the case of collective dynamics in multi-component liquids (with the number of species more than two) the theoretical and simulation studies turn out to be very scarce. Even the simplest dynamic properties like the relation between self- and mutual diffusion coefficients are not well defined, while the mechanism of formation of collective excitations and their contributions to the total spectral functions of three-component liquids have not been studied at all. Concerning ternary liquids, there were no reports on classification of propagating and relaxing modes beyond hydrodynamic region. Therefore, the main purpose of our study was to obtain dynamical eigenmodes in a simple Lennard-Jones three-component mixture using the parameter-free GCM approach as well as our previous GCM results on binary liquids in order to establish the origin of mode formation for different branches of propagating and relaxing excitations.

The paper is organized as follows. In section II an application of the method of generalized collective modes to the three-component Lennard-Jones liquid mixture is reported. In this study we follow the GCM scheme developed for multi-component fluids in [10]. An analytical treatment of the longitudinal and transverse mass-concentration fluctuations within some simplified dynamical models is presented in section III, and Section IV contains conclusions of this study.

\section{Static and time correlation functions}

\subsection{Details of methodology}

We have performed MD simulations for a three-component Lennard-Jones liquid $\mathrm{A}_{x} \mathrm{~B}_{y} \mathrm{C}_{y}$ with molar ratio 2:1:1 and mass ratio 13.91:8.63:4.63 at temperature $T=116 \mathrm{~K}$ and density $n=$ $0.0182 \AA^{-3}$. Parameters of LJ potentials were taken just as for the atoms Kr:Ar:Ar - hence, such a three-component system corresponds in the case of mass ratio 13.91:6.63:6.63 to a well-studied, within the GCM approach, equimolar KrAr liquid mixture [8,11]. Such a correspondence between the two systems was intentionally set up, because it permits to check most static averages and generalized thermodynamic quantities of the ternary liquid mixture with the ones of the binary Lennard-Jones KrAr liquid.

The time evolution of basis dynamical variables has been obtained from production runs of standard molecular dynamics simulations in microcanonical ensemble for the system of 2000 particles in a cubic box. Regular production run took over $3 \cdot 10^{5}$ time steps, while for five lowest $k$-values, in order to obtain the desired convergence of relevant static averages and time correlation functions, the system was simulated over $9 \cdot 10^{5}$ time steps. Twenty two $k$-points were sampled in MD simulations, and the smallest wavenumber reached in our MD study was $k_{\min }=0.131 \AA^{-1}$. Additional averages were taken over all the possible directions of wavenumbers corresponding to the same absolute value. In order to reduce the dimension of relevant quantities, the following energy, mass, spatial and time scales were used in our simulations: $\epsilon=k_{B} T, \mu=\bar{m}, \sigma=k_{\text {min }}^{-1}$, $\tau=\sigma(\mu / \varepsilon)^{1 / 2}=6.1056$ ps.

We combined MD simulations with the parameter-free GCM approach to study the longitudinal and transverse collective mode spectra, time correlation functions (TCFs) and separated contributions to certain TCFs, caused by various collective excitations and relaxation processes. For each sampled $k$-point, the shape of MD-derived time correlation functions and the spectrum of longitudinal eigenvalues were analyzed within the eleven-variable $\left(\mathrm{N}_{\mathrm{v}}=11\right) \mathrm{GCM}$ approach with 
the following basis set of dynamical variables:

$$
\mathbf{A}^{(11)}(k, t)=\left\{n_{A}, n_{\mathrm{B}}, n_{C}, J_{A}, J_{\mathrm{B}}, J_{C}, \varepsilon, \dot{J}_{A}, \dot{J}_{\mathrm{B}}, \dot{J}_{C}, \dot{\varepsilon}\right\},
$$

where the dynamical variables of partial densities are defined by

$$
n_{\alpha}(k, t)=\frac{1}{\sqrt{N}} \sum_{j=1}^{N_{\alpha}} \mathrm{e}^{-\mathrm{i} \mathbf{k r}_{j}^{\alpha}(t)}, \quad \alpha=A, B, C,
$$

the partial densities of longitudinal mass-currents are given by

$$
J_{\alpha}(k, t)=\frac{m_{\alpha}}{k} \frac{1}{\sqrt{N}} \sum_{j=1}^{N_{\alpha}}\left(\mathbf{k v}_{j}^{\alpha}\right) \mathrm{e}^{-\mathrm{i} \mathbf{k} \mathbf{r}_{j}^{\alpha}(t)} \quad \alpha=A, B, C,
$$

and density of total energy is

$$
\varepsilon(k, t)=\frac{1}{\sqrt{N}} \sum_{\alpha} \sum_{j=1}^{N_{\alpha}} e_{j}^{\alpha}(t) \mathrm{e}^{-\mathrm{ik \mathbf {kr } _ { j } ^ { \alpha }}(t)}
$$

Here the $e_{j}^{\alpha}(t)$ are single-particle energies in the $\alpha$ th species, estimated in a simple way as a sum of kinetic and potential energy of the $j$-th particle. In (1) and (3) we have dropped the label indicating the longitudinal component of partial mass-currents. One can obtain the first time derivatives of partial mass-currents and energy straightforwardly from the expressions (3) and (4).

In the case of transverse dynamics, the same level of treatment of short-time processes corresponds to a six-variable $\left(\mathrm{N}_{\mathrm{v}}=6\right)$ basis set of transverse dynamical variables, namely:

$$
\mathbf{A}^{(6 \mathrm{~T})}(k, t)=\left\{J_{A}^{\mathrm{T}}, J_{\mathrm{B}}^{\mathrm{T}}, J_{C}^{\mathrm{T}}, \dot{J}_{A}^{\mathrm{T}}, \dot{J}_{\mathrm{B}}^{\mathrm{T}}, \dot{J}_{C}^{\mathrm{T}}\right\} .
$$

Here the index $\mathrm{T}$ denotes the transverse component of currents and their time derivatives.

We would like to notice that the hydrodynamic set of dynamic variables for the ternary liquid mixtures includes

$$
\mathbf{A}^{(\text {hyd })}(k, t)=\left\{n_{A}, n_{\mathrm{B}}, n_{C}, J_{\mathrm{t}}, \varepsilon\right\}, \quad \mathbf{A}^{(\mathrm{hyd}, \mathrm{T})}(k)=\left\{J_{\mathrm{t}}^{\mathrm{T}}\right\}
$$

where longitudinal and transverse components of total mass-current $\mathbf{J}_{\mathrm{t}}(k, t)$ in the above adopted normalization (3) can be simply written in terms of partial mass-currents,

$$
\mathbf{J}_{\mathrm{t}}(k, t)=\mathbf{J}_{A}(k, t)+\mathbf{J}_{\mathrm{B}}(k, t)+\mathbf{J}_{C}(k, t) .
$$

Comparing (1) with the standard set of the hydrodynamic variables, one can see that within the GCM approach the partial density fluctuations are treated with the same precision in respect to the order of frequency sum rules reproduced explicitly as total density fluctuations. This is connected with the fact observed previously for binary liquids in the GCM treatment [8] and the numerical analysis of MD data [12] that some branches of propagating collective excitations have a pronouncedly partial character in the short-wavelength region and can be simply obtained from the analysis of MD-derived partial current-current spectral functions. Hence, the simplest extension of the hydrodynamic sets (6) by taking into account just the first time derivatives of hydrodynamic variables would not permit to correctly describe collective excitations in short-wavelength region. Therefore, all the partial currents are equitably considered in our theoretical scheme. Hereafter we shall call the TCFs, constructed on the hydrodynamic variables (6), the hydrodynamic time correlation functions.

All the static and time correlation functions, needed for the estimation of matrix elements of the $\mathrm{N}_{\mathrm{v}} \times \mathrm{N}_{\mathrm{v}}$ matrices of time correlation functions $\mathbf{F}(k, t)$ and their Laplace transforms $\tilde{\mathbf{F}}(k, z)$, 
were directly evaluated in computer simulations. Eigenvalues and eigenvectors of generalized hydrodynamic (otherwise referred to as kinetic) matrix [5]

$$
\mathbf{T}(k)=\mathbf{F}(k, 0) \cdot \tilde{\mathbf{F}}^{-1}(k, 0),
$$

were calculated for either of the twenty two $k$-points, sampled in MD. From the eigenvalues the spectra of propagating collective excitations were constructed and the $k$-dependent damping coefficients for the main relaxation processes were found. The eigenvectors were used in the analysis of mode contributions to time correlation functions of interest in different regions of wavenumbers.

\subsection{Generalized thermodynamic quantities}

The generalized hydrodynamic theory usually operates with the so-called generalized thermodynamic quantities (see, e.g., $[13,14]$ ), being $k$-dependent functions that tend in the limit $k \rightarrow 0$ to their thermodynamic values observed in real experiments. Here we follow the general expressions for the generalized thermodynamic quantities, obtained for $\nu$-component mixtures in [10]. In the case of a ternary liquid one has:

(i) for the generalized isothermal compressibility $\theta(k)$,

$$
\theta(k)=\frac{1}{n k_{\mathrm{B}} T}\left[\sum_{\alpha, \beta=1}^{3} c_{\alpha} F_{\alpha \beta}^{-1}(k) c_{\beta}\right]^{-1},
$$

where $n=N / V, c_{\alpha}$ is the concentration of particles in the $\alpha$-th species, and elements of the $3 \times 3$ matrix of partial density-density correlations are

$$
F_{\alpha \beta}(k)=\left\langle n_{\alpha}(k, 0) n_{\beta}^{*}(k, 0)\right\rangle ;
$$

(ii) for the generalized specific heat $C_{\mathrm{V}}(k)$ at constant volume per one particle,

$$
C_{\mathrm{V}}(k)=\frac{1}{k_{\mathrm{B}} T^{2}}\left\langle h(k, 0) h^{*}(k, 0)\right\rangle,
$$

where $h(k, t)$ is defined as follows

$$
h(k, t)=\varepsilon(k, t)-\sum_{\alpha, \beta=1}^{3}\left\langle\varepsilon(k, 0) n_{\alpha}^{*}(k, 0)\right\rangle F_{\alpha \beta}^{-1}(k) n_{\beta}(k, t) ;
$$

(iii) for the generalized linear thermal expansion coefficient $\alpha_{P}(k)$,

$$
\alpha_{P}(k)=\frac{1}{\mathrm{i} k}\left\langle\dot{\mathrm{t}}_{\mathrm{t}}(k, 0) h^{*}(k, 0)\right\rangle \frac{n \theta(k)}{k_{\mathrm{B}} T^{2}},
$$

where the static average of longitudinal stress-heat density correlation can be straightforwardly calculated from the definition (9);

(iv) for the generalized ratio of specific heats $\gamma(k)$,

$$
\gamma(k)=1+\frac{T \alpha_{P}^{2}(k)}{n \theta(k) C_{\mathrm{V}}(k)} .
$$

Numerical results, obtained directly in MD simulations as equal time averages, for the generalized thermodynamic quantities are shown in figure 1. Note, first, that at least three out of four generalized thermodynamic quantities in figure 1 have well pronounced peaks at $k$ nearby the position $k_{p} \simeq 1.8 \AA^{-1}$ of the first peak of the total static structure factor. Besides, some useful information can be extracted from such $k$-dependent thermodynamic quantities. In particular, from 


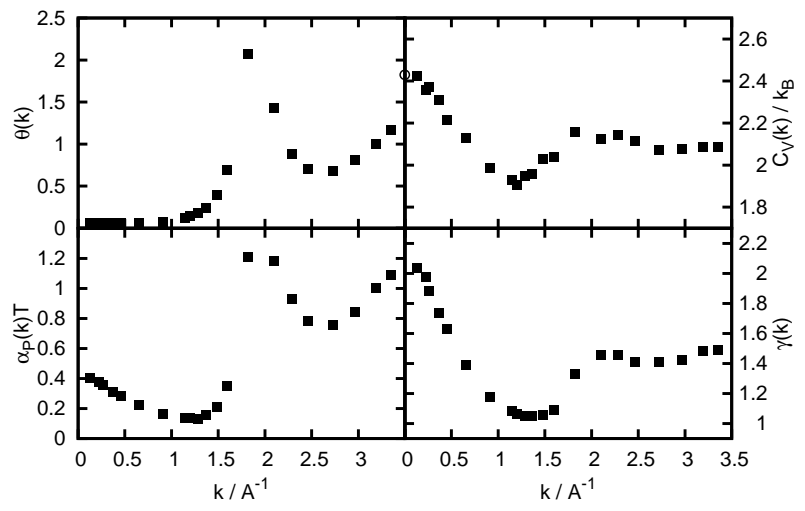

Figure 1. Generalized $k$-dependent thermodynamic quantities: generalized isothermal compressibility $\theta(k)$, generalized linear thermal expansion coefficient $\alpha_{P}(k)$, generalized specific heat at constant volume $C_{\mathrm{V}}(k)$, and generalized ratio of specific heats $\gamma(k)$. Open circle at $k=0$ in the upper right frame corresponds to specific heat at a constant volume obtained from the fluctuation formula during the MD run.

these calculations, considering small $k$ limit, one can predict the value of $\alpha_{\mathrm{T}}=0.0043 \mathrm{~K}^{-1}$ for linear expansion coefficient of such a ternary liquid at the temperature of $116 \mathrm{~K}$.

In the long-wavelength limit, the generalized $k$-dependent specific heat at constant volume $C_{\mathrm{V}}(k)$ is in agreement with the value of $2.43 k_{\mathrm{B}}$, independently obtained at $k=0$ via the fluctuation formula during the MD production runs. This value is quite reasonable for Lennard-Jones liquids. One should also note, that $\gamma(k)$ is in fact a measure of coupling between the heat and viscous processes: for $\gamma=1$ one can describe heat and viscous processes separately. That is why the longitudinal dynamics of liquid metals with the typical value of $\gamma$ being at the level of $1.1-1.2$ is described pretty well within the viscoelastic theories. For Lennard-Jones liquids the coupling between the heat and viscous processes is usually rather strong and, for instance, in our case only in the restricted range of wavenumbers nearby $k \simeq 1.3 \AA^{-1}$ the generalized ratio of specific heats $\gamma(k)$ is close to unity, so that the viscoelastic treatment could be successfully applied in this region.

\subsection{Time correlation functions}

In figure 2 the numerical results, obtained at $k=0.371 \AA^{-1}$ for time correlation functions describing the longitudinal dynamics of the three-component Lennard-Jones liquid in MD simulations, are presented. In the same figure we show our results for these TCFs found within the parameter-free GCM approach. Hence, one can see how the GCM approach makes it possible to reproduce the main TCFs of primary interest obtained in MD simulations. Note that the time scale of $\tau$ indicates that the range of time correlations at $k=0.37 \AA^{-1}$ is of the order of 70 ps. For this reason, to provide good convergence of the tails of time correlation functions, we have simulated the system three times longer for the smallest wavenumbers.

The GCM replicas were calculated using theoretical expressions for time correlation functions (see, e.g., [5]), which in this study for the case of basis set (1) could be represented as linear combinations of the eleven contributions from the dynamical eigenvalues that correspond either to generalized collective propagating excitations or $k$-dependent relaxation processes, namely

$$
\mathrm{F}_{i j}^{(\mathrm{GCM})}(k, t)=\sum_{\alpha=1}^{\mathrm{N}_{\mathrm{v}}} \mathrm{G}_{\alpha}^{i j}(k) \mathrm{e}^{-z_{\alpha}(k) t},
$$

where $\mathrm{G}_{\alpha}^{i j}(k)$ are the $k$-dependent amplitudes, describing the corresponding partial contribution of the collective mode $z_{\alpha}(k)$ to the shape of $\mathrm{F}_{i j}(k, t)$. Both the amplitudes $G_{\alpha}^{i j}(k)$ and the eigenvalues $z_{\alpha}(k)$ can be in general the complex quantities and are calculated from the 


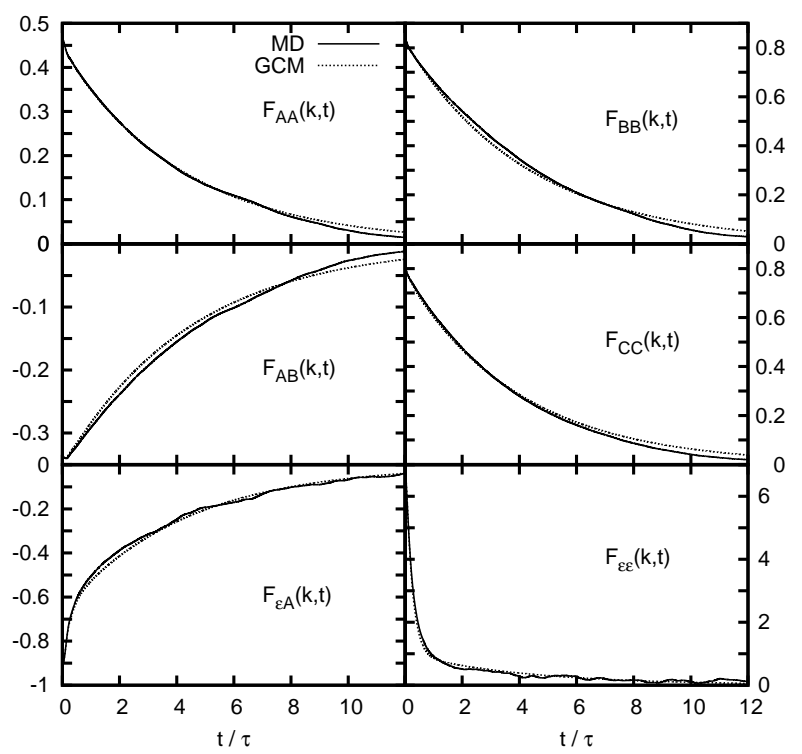

Figure 2. Partial density-density, density-energy and energy-energy time correlation functions Fij $(k, t)$, obtained in MD simulations (solid lines) and their theoretical replicas (dotted lines), calculated within the parameter-free GCM approach for $k=0.371 \AA^{-1}$. For partial TCFs a normalization constant was used in order to correspond to Ashcroft-Langreth partial structure factors at $t=0$. The time scale is $\tau=6.1056 \mathrm{ps}$.

eigenvalue problem for the generalized hydrodynamic matrix (8), generated in our case on the eleven-variable basis set $\mathbf{A}^{(11)}(k)$.

Comparing the theoretical curves with MD data, shown in figure 2, it is seen that the GCM approach permits to reproduce the time-dependence of MD-derived TCFs with very good precision without any fitting parameters. We stress that for each wavenumber the obtained set of eleven $\left(\mathrm{N}_{\mathrm{v}}=11\right)$ dynamical eigenvalues and relevant eigenvectors reproduce simultaneously $\mathrm{N}_{\mathrm{v}}\left(\mathrm{N}_{\mathrm{v}}+\right.$ 1)/2 time correlation functions. Moreover, the TCFs of primary interest, describing the density fluctuations, are reproduced within the GCM scheme used in this study with the precision that the sum rules are satisfied explicitly up to the fourth order inclusive.

Using the TCFs shown in figure 2, one can define the main correlation times of a ternary liquid as follows:

$$
\tau_{i j}(k)=\frac{1}{\mathrm{~F}_{i j}(k, 0)} \int_{0}^{\infty} \mathrm{F}_{i j}(k, t) \mathrm{d} t, \quad i, j=A, B, C, \varepsilon .
$$

In general, in the case of a ternary mixture one can find ten independent correlation functions, which in this study were directly estimated from MD-derived TCFs and were subsequently used in the calculation of the matrix elements of generalized hydrodynamic matrix $\mathbf{T}(k)$, generated on the basis set $\mathbf{A}^{(11)}(k, t)$.

\section{Spectra of collective excitations}

\subsection{Dispersion and damping coefficients for transverse and longitudinal excitations}

Let us start from the discussion of transverse dynamics, because due to the absence of coupling with the heat fluctuations, this case turns out to be much simpler for the analysis than the longitudinal dynamics. In figure 3 we report the imaginary and real parts of complex eigenvalues

$$
z_{\alpha}^{\mathrm{T}}(k)=\sigma_{\alpha}^{\mathrm{T}}(k)+\mathrm{i} \omega_{\alpha}^{\mathrm{T}}(k),
$$


which correspond to the dispersion and damping of propagating collective modes, respectively. Purely real transverse eigenvalues

$$
\operatorname{Im} z_{j}^{\mathrm{T}}(k)=0, \quad \operatorname{Re} z_{j}^{\mathrm{T}}(k)=d_{j}^{\mathrm{T}}(k)
$$

will be marked by symbols $d_{j}^{\mathrm{T}}(k)$. They have the meaning of inverse $k$-dependent lifetimes of the relevant relaxation processes. We stress that our approach provides a straightforward and consistent extension of the standard hydrodynamics that allows us to take into account the $k$-dependent relaxation processes of non-hydrodynamic origin. Like in the case of hydrodynamic processes, the non-hydrodynamic relaxation processes make exponential contributions with $k$-dependent amplitudes to the shape of the relevant hydrodynamic time correlation functions.

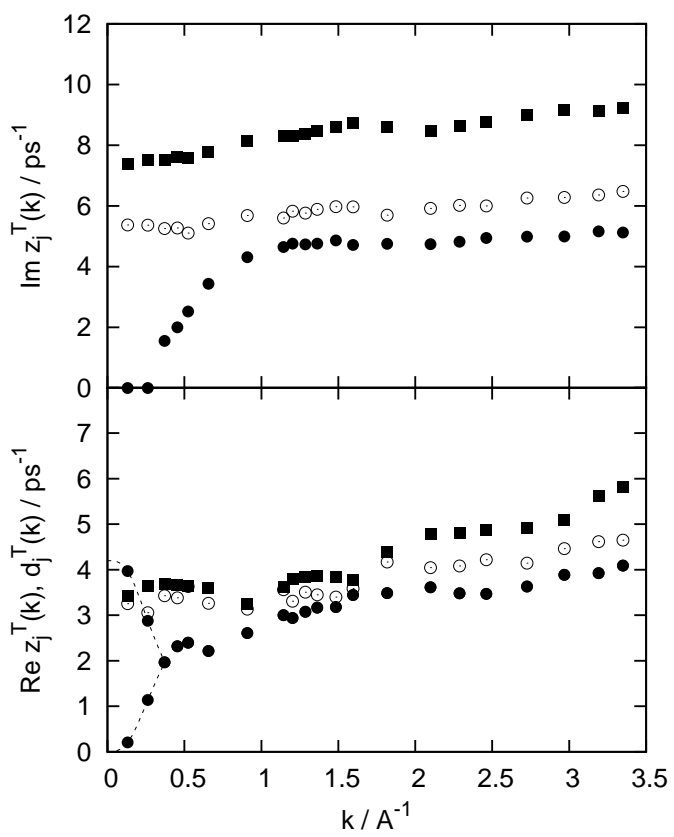

Figure 3. Imaginary (top) and real (bottom) parts of complex eigenvalues for transverse collective excitations in a wide region of wavenumbers. In the lower frame the real parts of complex eigenvalues are shown together with the purely real eigenvalues $d_{j}^{\mathrm{T}}(k)$, describing the relaxation processes in transverse dynamics. Dashed lines in small $k$ range are spline-interpolation for purely real eigenvalues.

In figure 3, purely real eigenvalues are shown by dash-line connected symbols. It is seen that these eigenvalues occur only in a narrow region for $k<0.4 \AA^{-1}$. The slowest transverse relaxation process with the smallest eigenvalue, $d_{1}^{\mathrm{T}}(k)$, gives an infinite relaxation time in the $k \rightarrow 0$ limit and according to the hydrodynamics should behave like

$$
d_{1}^{\mathrm{T}}(k)=\frac{\eta}{\rho} k^{2}, \quad \text { when } \quad k \rightarrow 0
$$

with $\eta$ and $\rho$ being the shear viscosity and mass density of the mixture, respectively. The estimated value of the shear viscosity is $\eta=2.24 \cdot 10^{-4} \mathrm{~kg} / \mathrm{s} \cdot \mathrm{m}$. The second type of relaxation process, seen in figure 3, is of non-hydrodynamic origin and describes the process with a finite lifetime in the long-wavelength limit, defined mainly by the rigidity modulus $G$ (see also [1])

$$
d_{2}^{\mathrm{T}}(k)=\frac{G}{\eta}-\frac{\eta}{\rho} k^{2}, \quad k \rightarrow 0 .
$$

For larger $k, k>0.4 \AA^{-1}$, both relaxing modes merge and the pair of propagating collective modes known as shear waves emerge in the system. Thus, the both relaxing modes $d_{j}^{\mathrm{T}}(k)$ and the 
emergence of shear waves are in complete analogy with the results known so far for simple and binary liquids.

The difference in the transverse dynamics of a ternary mixture in comparison with one- and twocomponent cases appears in the number of high-frequency propagating modes. In the case of threecomponent liquid mixture within the six-variable GCM treatment we have found two branches of high-frequency transverse propagating modes existing in the whole $k$-range considered. In figure 3 one can see the main features of transverse propagating modes in a wide range of wavenumbers: (i) two high-frequency branches with rather close damping coefficients tend to finite frequencies in the long-wavelength limit, which implies their origin as optic phonon-like excitations; (ii) all three branches of propagating modes, obtained in our study, have very close damping coefficients in the region of $1 \AA^{-1}<k<1.8 \AA^{-1}$; (iii) a legible separation in frequency and damping coefficients is observed for all three propagating modes in short-wavelength region $k>2 \AA^{-1}$. The origin of high-frequency branches of transverse excitations will be discussed in the next subsection.

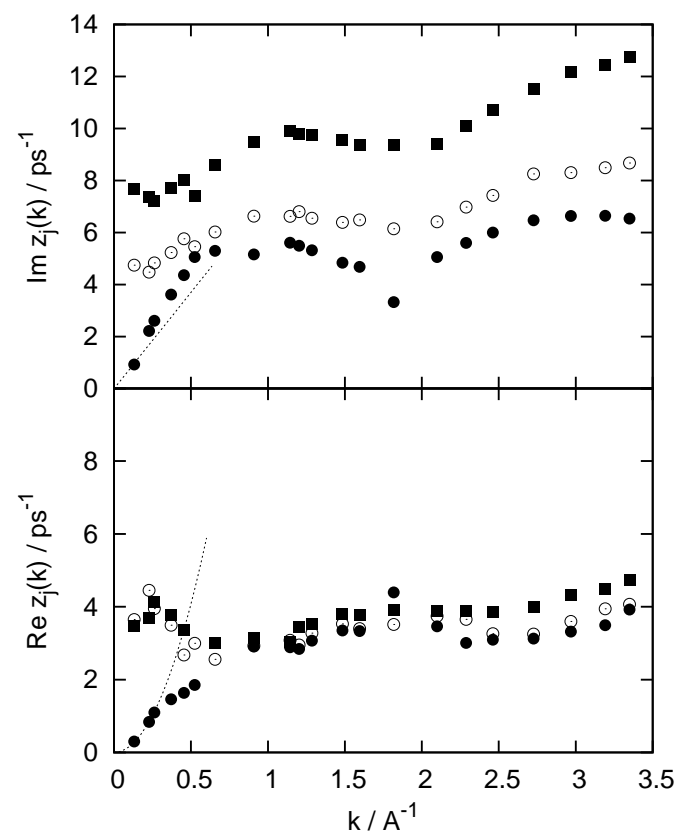

Figure 4. Imaginary (top) and real (bottom) parts of the longitudinal complex eigenvalues, obtained on the eleven-variable basis set $\mathbf{A}^{(11)}$.

In figure 4, the dispersion and damping coefficients of longitudinal propagating modes, obtained within the same level of treatment of short-time processes as in the transverse case, are shown. In the whole range of wavenumbers, using the set (1), we have found three branches of propagating modes (presented in figure 4). Moreover, for $k>1 \AA^{-1}$ there can appear an additional pair of propagating excitations that correspond to low-frequency heat waves (not shown in figure 4 ). The three branches of propagating excitations shown in figure 4 can be easily identified only in longwavelength region, where the lowest branch behaves like the longitudinal sound modes having dispersion $\omega(k)$ with obvious positive deviation (positive dispersion) from linear dependence and the damping coefficient being proportional to $k^{2}$, as is shown by relevant dotted lines in the small $k$ domain of a lower frame in figure 4 . The estimated values of sound velocity and damping coefficient are $c=704 \mathrm{~m} / \mathrm{s}$ and $\Gamma=1.7 \cdot 10^{-7} \mathrm{~m}^{2} / \mathrm{s}$, respectively. For a larger $k, k>0.7 \AA^{-1}$, all three branches have comparable damping, while the frequencies are still well separated. The origin of the other two branches will be discussed more in detail herein below, where an analysis on some simplified models of the longitudinal dynamics is presented.

Purely real eigenvalues, obtained from the description of the longitudinal dynamics within the eleven-variable dynamical model (1), are shown in figure 5 for small wavenumbers $k$. One can easily 


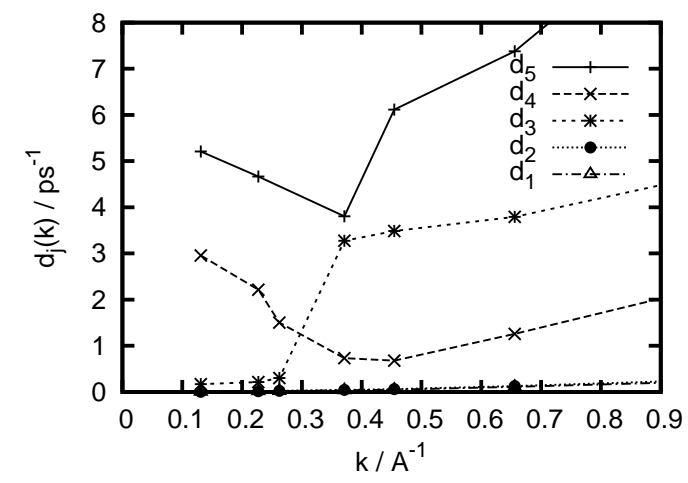

Figure 5. Longitudinal purely real eigenvalues, obtained within the eleven-variable GCM treatment in long-wavelength region.

see two kinetic relaxation modes, which tend to nonzero constants when $k \rightarrow 0$, while the damping coefficients for hydrodynamic relaxation processes should behave like $k^{2}$ in the long-wavelength limit. In our case there exist two heat relaxing modes: (i) hydrodynamic one (shown as $d_{3}(k)$ in figure 5) that displays the standard $k$-dependence

$$
d_{3}(k)=D_{\mathrm{T}} k^{2}, \quad \text { when } \quad k \rightarrow 0,
$$

and (ii) kinetic heat relaxing mode (marked as $d_{5}(k)$ in figure 5), which according to [7] should behave (without taking into account the coupling with the viscous processes) approximately like

$$
d_{5}(k)=\frac{n G^{\mathrm{h}}}{m D_{\mathrm{T}}}-D_{\mathrm{T}} k^{2}, \quad \text { when } \quad k \rightarrow 0,
$$

where $G^{\mathrm{h}}$ is a heat rigidity modulus.

Another kinetic relaxing mode $d_{4}(k)$, which has a finite lifetime in long-wavelength limit and decays much faster in time in comparison with the hydrodynamic relaxing processes, is of viscoelastic origin and according to [15] behaves in the long-wavelength limit roughly as

$$
d_{4}(k)=\frac{c_{\infty}^{2}-c_{s}^{2}}{D_{\mathrm{L}}}-D_{\mathrm{L}} k^{2},
$$

where $c_{s}, c_{\infty}$ are adiabatic and infinite-frequency sound velocities, respectively, and $D_{\mathrm{L}}$ is a kinematic viscosity.

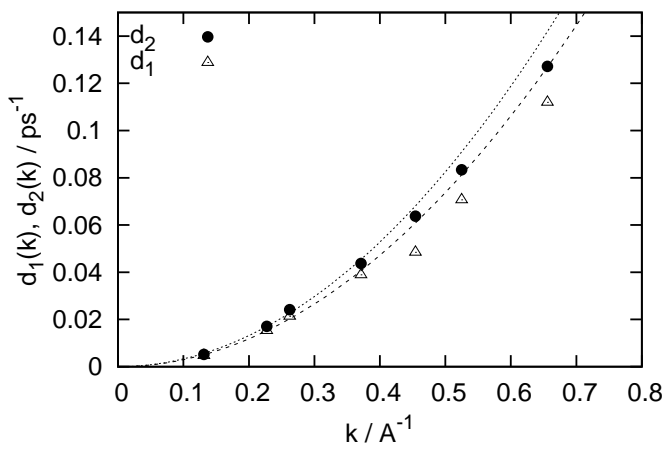

Figure 6. Long-wavelength asymptotes of the two lowest real eigenvalues corresponding to the relaxation processes of mutual diffusivity.

The two lowest relaxing modes $d_{1}(k)$ and $d_{2}(k)$ in small $k$ domain describe the relaxation processes connected with mutual diffusion of species. In figure 6 their long-wavelength behavior 
with hydrodynamic asymptotes proportional to $k^{2}$ is shown. From this figure one can see that for both diffusive modes there exists a negative deviation from the hydrodynamic asymptotes when $k$ increases. This corresponds to a decrease with $k$ of the generalized $k$-dependent mutual diffusion coefficients $D_{x 1}(k)$ and $D_{x 2}(k)$, which is known from our previous studies of generalized transport coefficients in binary liquids [16]. From these asymptotes we can easily estimate the corresponding values of damping coefficients for the ternary mixture studied: $D_{x 1}=2.75 \cdot 10^{-9} \mathrm{~m}^{2} / \mathrm{s}$ and $D_{x 2}=3.1 \cdot 10^{-9} \mathrm{~m}^{2} / \mathrm{s}$. In general, one can say that the heat relaxing processes for $k>0.4 \AA^{-1}$ have much smaller lifetime comparing with the viscoelastic processes that reflect the structural relaxation phenomena mainly in intermediate and short-wavelength region.

\subsection{Simplified models of transverse dynamics}

In order to study more in detail the origin of mode formation for different branches in the spectrum of collective excitations we proceed as in our previous GCM studies of collective dynamics in simple and binary liquids. Namely, let us try to find the separated subsets of dynamical variables, which would reproduce well the spectra (or its parts) of eigenvalues in some regions of wavenumbers. In such a case one can suppose that the coupling between the actual subset and other dynamical variables is really small and can be neglected. Similar approach allows us to identify the heat waves in liquid metals [7] and optic-like excitations in binary liquids [8,9].

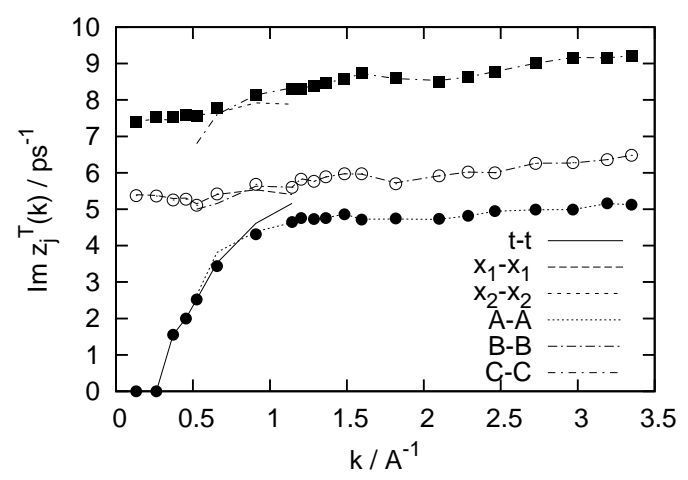

Figure 7. Imaginary parts of the transverse complex-conjugated eigenvalues, obtained for the six-variable basis set $\mathbf{A}^{(6 \mathrm{~T})}$ (symbols) and calculated on the separated subsets of dynamical variables (lines) $\mathbf{A}_{\mathrm{i}}^{(2 \mathrm{~T})}$ and $\mathbf{A}_{\alpha}^{(2 \mathrm{~T})}$ for small and intermediate $k$ values, respectively.

Let us split the six-variable dynamical set (5), used for the case of transverse dynamics, into three separated two-variable dynamical subsets, describing collective transverse processes only within the species:

$$
\mathbf{A}_{\alpha}^{(2 \mathrm{~T})}(k, t)=\left\{J_{\alpha}^{\mathrm{T}}, \dot{J}_{\alpha}^{\mathrm{T}}\right\}, \quad \alpha=A, B, C .
$$

Then we have used these subsets in order to generate three different $2 \times 2$ generalized kinetic matrices $\mathbf{T}_{\alpha}^{(2 \mathrm{~T})}(k)$. This resulted in three complex-conjugated pairs of eigenvalues shown in figure 7 by corresponding lines for $k>0.5 \AA^{-1}$. One can see that the results obtained on the separated subsets, describing partial dynamics, match perfectly with the results found for the six-variable dynamical model. This means that the partial dynamics dominates in this region, $k>0.8 \AA^{-1}$, and therefore we conclude that the collective dynamics becomes essentially of "partial" type and can be well described in terms of "partial" branches of collective excitations for larger $k$. For smaller wavenumbers, the results obtained for the two-variable dynamical models (11) differ essentially from the results found for the general six-variable dynamical model (5). Hence, it is obvious that in a small $k$ domain, the collective dynamics is determined by another type of collective behavior.

In the case of binary liquids it was rather easy to construct other subsets of transverse dynamical variables, reflecting collective properties: it was obvious that one of them should be based on the total transverse mass-current (the hydrodynamic variable), while another subset was constructed 
for the variable being orthogonal to the total mass-current as a combination of partial currents. In the case of a ternary mixture we have proceeded in a similar manner and found two dynamical variables being orthogonal between themselves as well as to the total mass-current. They can be defined as follows

$$
J_{x 1}^{\mathrm{T}}(k, t)=x_{A} J_{B}^{\mathrm{T}}(k, t)-x_{B} J_{A}^{\mathrm{T}}(k, t)
$$

and

$$
J_{x 2}^{\mathrm{T}}(k, t)=\left(x_{A}+x_{B}\right) J_{C}^{\mathrm{T}}(k, t)-x_{C}\left(J_{A}^{\mathrm{T}}(k, t)+J_{B}^{\mathrm{T}}(k, t)\right),
$$

where the dynamical variable $J_{x 1}^{\mathrm{T}}(k, t)$ describes the transverse component of mutual current of the most heavy and medium-weight components, while the dynamical variable (13) corresponds to mutual current of the most light component against two heavier ones.

On this basis we have considered another three separated subsets of dynamical variables

$$
\mathbf{A}_{\mathrm{i}}^{(2 \mathrm{~T})}(k, t)=\left\{J_{\mathrm{i}}^{\mathrm{T}}, \dot{J}_{\mathrm{i}}^{\mathrm{T}}\right\}, \quad \mathrm{i}=t, x 1, x 2,
$$

and performed new calculations of collective mode spectra. This allows us to reproduce very nicely and separately all the branches of propagating collective excitations in long-wavelength region. The results are shown in figure 7 by corresponding lines in small $k$ domain. Hence, by analogy with the case of binary liquids we conclude that both high-frequency branches of kinetic propagating modes correspond to optic-like excitations, formed by fluctuations of mutual mass-currents (12) and (13). Since the three subsets (14) describe intrinsic collective fluctuations, one may refer to the long-wavelength region as the region of coherent dynamics, just as pointed out by Hafner in 1983 in the study of a binary magnetic glass [17].

\subsection{Simplified models of longitudinal dynamics}

The analysis of longitudinal spectrum is much more sophisticated due to the coupling between viscoelastic and thermal fluctuations. Let us start our consideration from the simplest dynamical models, based on three-variable subsets that consist of the partial dynamical variables only:

$$
\mathbf{A}_{\alpha}^{(3)}(k, t)=\left\{n_{\alpha}, J_{\alpha}, \dot{J}_{\alpha}\right\}, \quad \alpha=A, B, C .
$$

All these models result in a relaxing mode and in a pair of propagating collective excitations with complex-conjugated eigenvalues, shown in the lower frame of figure 8 by corresponding lines. Once again, comparing with the results found for the eleven-variable model in the short-wavelength region, we see that all three models of partial dynamics permit to reproduce perfectly and separately all the propagating collective excitations. In the opposite range of small $k$ they produce the dispersion laws that tend to relevant nonzero frequencies at $k \rightarrow 0$. Comparing with the results found for a more sophisticated model (1) we note that the dispersion laws for the upper lying modes are rather well reproduced up to the smallest $k$ reached in our study, while for the lower pair of propagating modes one can see a significant deviation in small $k$ domain. Thus, in complete agreement with the results found for the transverse dynamics, we conclude that the "partial" type of collective behavior dominates in the range of intermediate and large wavenumbers, $k>0.8 \AA^{-1}$, and determine the dynamical properties of the mixture in this region.

In order to separately describe the branches of longitudinal collective excitations in the region of "coherent" dynamics let us consider another class of simplified dynamic models that consists of the following basic variables:

(i) the total density

$$
n_{\mathrm{t}}(k, t)=n_{A}(k, t)+n_{B}(k, t)+n_{C}(k, t) ;
$$

(ii) two mass-concentration densities

$$
n_{x 1}(k, t)=\frac{m_{A}}{\bar{m}} x_{B} n_{A}(k, t)-\frac{m_{B}}{\bar{m}} x_{A} n_{B}(k, t),
$$




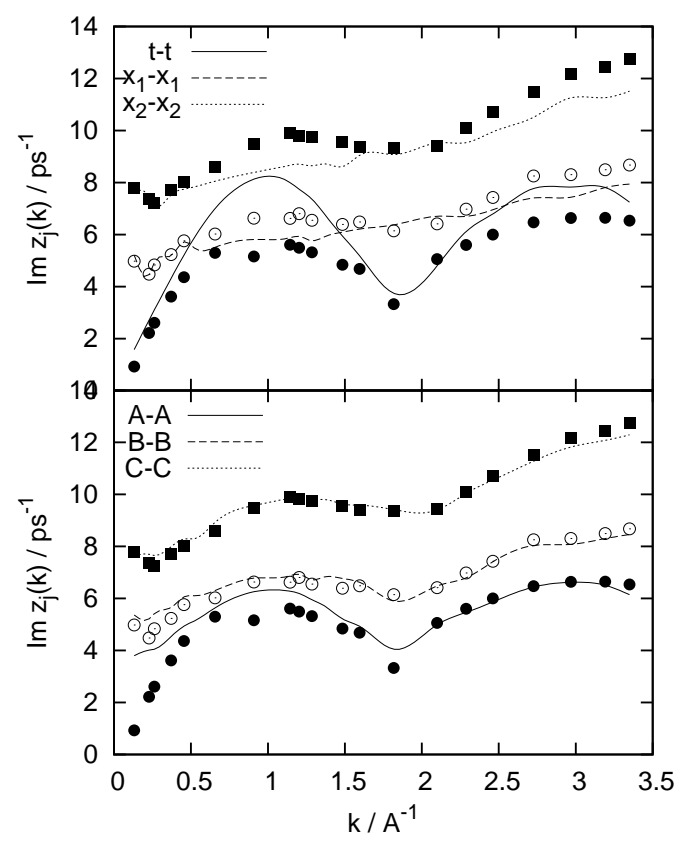

Figure 8. Imaginary parts of the longitudinal complex-conjugated eigenvalues, obtained for the eleven-variable basis set $\mathbf{A}^{(11)}$ (symbols) and calculated on the separated subsets of dynamical variables (lines) $\mathbf{A}_{\alpha}^{(3)}$ (bottom) and $\mathbf{A}_{\mathrm{i}}^{(3)}$ (top).

and

$$
n_{x 2}(k, t)=\frac{1}{\bar{m}}\left\{\left(x_{A}+x_{B}\right) m_{C} n_{C}(k, t)-x_{C}\left[m_{A} n_{A}(k, t)+m_{B} n_{B}(k, t)\right]\right\}
$$

being the linear combinations of partial densities. It is seen from (17) and (18) that the massconcentration densities have simple relation to the relevant longitudinal mass-concentration currents $J_{x i}(k, t)$, namely,

$$
\frac{\partial n_{x i}(k, t)}{\partial t}=\frac{i k}{\bar{m}} J_{x i}(k, t), \quad i=1,2
$$

constructed in the same manner as in the case of transverse dynamics (see (12) and (13)). It is easy to check that both mass-concentration currents $J_{x}(k, t)$ are mutually orthogonal dynamic variables and both are orthogonal to the total mass-current density $J_{t}(k, t)$. This makes the theoretical treatment of dynamical processes in small $k$ region especially convenient, where the "coherent" type of the dynamics prevails, because it allows us to perform the analysis in terms of $t-x$ variables (see [9]).

In the upper frame of figure 8 we show by corresponding lines the results for propagating collective modes, obtained within the simplified dynamical models that are defined on the separated subsets $\mathbf{A}_{\mathrm{i}}^{(3)}(k, t)$ with $\mathrm{i}=t, x 1, x 2$ (compare with (15)). One can see that for $k<0.5 \AA^{-1}$ the propagating modes, calculated for the subsets $\mathbf{A}_{\mathrm{x} 1}^{(3)}(k, t)$ and $\mathbf{A}_{\mathrm{x} 2}^{(3)}(k, t)$, perfectly reproduce both high-frequency longitudinal branches. This makes an evidence of the origin of these collective excitations as optic phonon-like modes. It is also seen in figure 8 that the low-frequency branch is well described in the long-wavelength region on the separated subset $\mathbf{A}_{\mathrm{t}}^{(3)}(k, t)$ which includes only the dynamical variables representing total mass-density and its two time derivatives (total current and longitudinal projection of stress tensor). We note that there exists a close analogy between the model $\mathbf{A}_{\mathrm{t}}^{(3)}$ and the so-called viscoelastic model of a fluid [1,2]. Besides one purely real eigenvalue, both these models result in the appearance of a pair of propagating sound modes with the linear $k$-dependent dispersion in long-wavelength limit with propagation velocity equal to $c_{\infty}$. Only the correct treatment of coupling with the heat fluctuations bends down the dispersion curve for sound 
modes in the long-wavelength region and changes the propagation velocity to the correct value $c_{s}$, known from the standard hydrodynamics.

We may conclude herein, that in both cases of the transverse and longitudinal dynamics our analysis indicates an evident crossover between the "coherent" and "partial" types of collective behavior in the ternary liquid mixture studied. It is also established that this crossover is observed in the region of $0.5 \AA^{-1}<k<1 \AA^{-1}$ in both cases.

\section{Conclusions}

We have performed the analysis of generalized collective mode spectra for a ternary LennardJones liquid mixture. A parameter-free GCM approach has been used to reach the goal of this study. Our main conclusions can be formulated as follows:

(i) For a ternary liquid mixture we have found two additional high-frequency optic-like propagating modes in both cases of the longitudinal and transverse dynamics. Within the GCM scheme used in this study the number of optic-like modes is simply related to the number of components in a multi-component mixture, and this has a close analogy with a solid-like picture;

(ii) Another specific feature in the longitudinal dynamics of a ternary mixture in comparison with a binary liquid is the appearance of an additional relaxation process connected with mutual diffusion, i.e. in the three-component liquid mixture we have observed two relaxation processes with different damping coefficients connected with diffusion coefficients;

(iii) Two linear combinations of partial currents have been found that permit to separately reproduce the optic-like modes of a ternary liquid in the long-wavelength limit. One of these two combinations describes mutual current of the most heavy and medium-weight components, while another one reflects mutual current of the most light component against two heavier ones;

(iv) An analysis, performed on the separated subsets of dynamical variables, permits us to establish a crossover from a "coherent" to "partial" type of collective dynamics in the dispersion laws of propagating collective modes. The "coherent" type of collective dynamics is observed in a small $k$ region and is represented by sound modes (in the transverse case - shear waves) and propagating optic-like excitations, while in the range of "partial" dynamics (intermediate and large $k$ ) the dynamical properties are determined mainly by "partial" motions of particles of each species;

(v) We have calculated the generalized thermodynamic quantities for the ternary liquid mixture. This seems to be the first example of such calculations performed for three-component liquids.

\section{Acknowledgements}

I.M. thanks the Fonds zur Förderung der wissenschaftlichen Forschung (Austria) for support under Project No P18592-TPH. 


\title{
References
}

1. Boon J.-P., Yip S. Molecular Hydrodynamics, New-York: McGraw-Hill, 1980.

2. Balucani U., Zoppi M. Dynamics of the liquid state, Oxford: Clarendon, 1983.

3. Chong S.-H., Hirata F., Phys. Rev. E, 1998, 57, 1691.

4. Chong S.-H., Hirata F., Phys. Rev. E, 1998, 58, 6188.

5. Mryglod I.M., Omelyan I.P., Tokarchuk M.V., Mol. Phys., 1995, 84, 235.

6. Mryglod I.M., Omelyan I.P., Mol. Phys., 1997, 90, 91.

7. Bryk T., Mryglod I., Phys. Rev. E, 2001, 63, 051202.

8. Bryk T., Mryglod I., J. Phys.: Cond. Matt., 2000, 12, 6063.

9. Bryk T., Mryglod I., J. Phys.: Cond. Matt., 2002, 14, L445.

10. Mryglod I.M., Condens. Matter Phys., 1997, No 10, 115.

11. Bryk T., Mryglod I., Condens. Matter Phys., 2004, 7, 15.

12. Anento N., Padro J.A., Phys. Rev. B, 2000, 62, 11428.

13. de Schepper I.M., Cohen E.G.D., Bruin C., van Rijs J.C., Montfrooij W., de Graaf L.A., Phys. Rev. A, 1988, 38, 271.

14. Bryk T., Mryglod I., Kahl G., Phys. Rev. E, 1997, 56, 2903.

15. Bryk T., Mryglod I., Condens. Matter Phys., 2004, 7, 471.

16. Bryk T., Mryglod I., Condens. Matter Phys., 2004, 7, 285.

17. Hafner J., J. Phys. C, 1983, 16, 5773.

\section{Динаміка потрійних рідких сумішей: аналіз узагальнених колективних мод}

\author{
Т.М.Брик ${ }^{1,2}$, І.М.Мриглод ${ }^{1,3}$ \\ 1 Інститут фізики конденсованих систем НАН України, 79011 Львів, вул. Свєнціцького, 1 \\ 2 Інститут прикладної математики і фундаментальних наук, \\ Національний університет “Львівська політехніка", 79013 Львів, Україна \\ 3 Інститут теоретичної фізики, Лінцський університет, 4040 Лінц, Австрія
}

Отримано 13 листопада 2007 р.

Підхід узагальнених колективних мод (УКМ), що не містить жодних вільних параметрів, використано для дослідження повздовжньої і поперечної динаміки потрійної рідкої Леннард-Джонсівської суміші. Розраховано спектри колективних збуджень у широкій області зміни значень хвильового вектора - від гідродинамічної і до області молекулярного режиму. Для з'ясування механізмів формування колективних збуджень, що спостерігаються у спектрах, використано кілька спрощених динамічних моделей. Проведений аналіз дозволив виявити кросовер від когерентної до парціальної динаміки у дисперсійних залежностях, отриманих для пропагаторних колективних збуджень. Когерентний тип динаміки спостерігається у гідродинамічній області і асоціюється із звуковими модами (зсувними хвилями у випадку поперечної динаміки) та пропагаторними збудженнями оптичного типу. В області парціальної динаміки (проміжні і великі значення хвильових векторів) динамічні властивості визначаються в основному скорельованими парціальними рухами частинок кожного із сортів. В рамках використаного підходу УКМ число пропагаторних збуджень оптичного типу, які $є$ ідентифіковані у цьому досліджені, визначається числом компонент у багатокомпонентній суміші, що має пряму аналогію у динаміці твердотільних систем.

Ключові слова: потрійні рідкі суміші, колективні збудження, моди оптичного типу, молекулярна динаміка

PACS: 05.20.Jj, 61.20.Ja, 61.20.Lc 